\title{
Formulation Optimization of Gentamicin Loaded Eudragit RS100 Microspheres Using Factorial Design Study
}

\author{
Deependra Singh, ${ }^{a}$ Swarnlata SARAF, ${ }^{a}$ Vinod Kumar DiXIt,${ }^{b}$ and Shailendra SARAF $* a$ \\ ${ }^{a}$ Institute of Pharmacy, Pt. Ravishankar Shukla University; Raipur, C.G.-492010, India: and ${ }^{b}$ Department of \\ Pharmaceutical sciences, Dr. H.S. Gour University; Sagar (M.P.), India.
}

Received July 17, 2007; accepted January 11, 2008; published online January 25, 2008

Gentamicin-Eudragit RS100 microspheres were prepared by modified double emulsion method. A $3^{2}$ full factorial experiment was designed to study the effects of the composition of outer aqueous phase in terms of amount of glycerol (viscosity effect) and sodium chloride (osmotic pressure gradient effect) on the entrapment efficiency and \% yield and microsphere size. The results of analysis of variance test for responses measured indicated that the test is significant $(p>0.05)$. The contribution of sodium chloride concentration was found to be higher on entrapment efficiency and \% yield, whereas glycerol produced significant effect on the mean diameter of microspheres. Microspheres demonstrated spherical particles in the size range of $33.24-60.43 \mu \mathrm{m}$. In vitro release profile of optimized formulation demonstrated sustained release for $\mathbf{2 4} \mathrm{h}$ following Higuchi kinetics. Finally, drug bioactivity was found to remain intact after microencapsulation. Response surface graphs are presented to examine the effects of independent variables on the responses studied. Thus, by formulation design important parameters affecting formulation characteristics of gentamicin loaded Eudragit RS100 microspheres can be identified for controlled delivery with desirable characters in terms of maximum entrapment and yield.

Key words gentamicin; factorial design; microsphere; response surface graph; Eudragit RS100

Wound healing is a complex multifactorial process that results in contraction and closure of wound and restoration of a functional barrier. ${ }^{1)}$ It is important to keep the wound free of infection eliminating all potential factors inhibiting natural healing process. ${ }^{2)}$ Antibiotics are effective against the microbes in vitro; however, during infection the bacterium is localized intracellularly making the treatment difficult. ${ }^{3)}$ Thus there is a need of delivery vehicles that allow localized and controlled delivery of antibiotics for preventing microbial infections by intracellular pathogens. ${ }^{4)}$

Particulate carriers such as microspheres may target the antibiotics to the intracellular sites where the bacterium is found, and as being in a sustained manner, would permit to reduce the number of doses and decrease drug toxicity. ${ }^{4)} \mathrm{A}$ variety of microencapsulation techniques are used for effective encapsulation of drugs. ${ }^{5,6)}$ Among these techniques, immobilization of drug in a hydrophobic polymer by either a non-aqueous phase separation or the evaporation of the volatile solvent of the internal phase of an oil in o/o emulsion has been proposed for encapsulation of water soluble drugs. ${ }^{6,7)}$ But, the double emulsion method (w/o/w) remains the widely acceptable alternative method for the encapsulation of hydrophilic drugs. ${ }^{8)}$ This method involves the dispersion of an aqueous solution of drug into an organic solution containing the polymer followed by emulsification. This primary emulsion (w/o) is then dispersed in a second aqueous phase containing a suitable emulsifier. Solid microspheres are collected following the complete removal of the volatile organic solvent.

Gentamicin sulphate is a hydrophilic aminoglycoside antibiotic with short half-life of $2-4 \mathrm{~h}$ used in the treatment of serious microbial infections. ${ }^{9}$ ) It has been reported that hydrophilic drugs tend to come out in the outer aqueous phase when microspheres are fabricated using double emulsion method, leading to decreased entrapment. ${ }^{10)}$ However, the encapsulation efficiency can be improved and the physicochemical properties of the microspheres such as the particle size, size distribution, surface texture, morphology and drug release profile can be controlled by altering the preparative conditions. ${ }^{11)}$ Kassas prepared Eudragit microspheres with increased entrapment of gentamicin by incorporating different concentration of $\mathrm{KCl}$ and gentamicin in the external aqueous phase. ${ }^{12)}$ However, quantitative aspects of the effects and relationship among various factors have not been studied yet.

The technique of factorial design is an efficient method indicating the relative significance of a number of variables and their interactions. Many researchers have applied it for dosage form designing of various drugs. ${ }^{13-15)}$

A $3^{2}$ factorial design is an established method to study the action of selected parameters. In the present study, we aimed to develop microparticulate system for controlled release of antimicrobial agent in the alkaline media as open wounds tend to have a neutral or alkaline $\mathrm{pH}$, predominantly in the range of $6.5-8.5 .^{16)}$ In the present investigation, the effect of Glycerol $\left(X_{1}\right)$ and $\mathrm{NaCl}\left(X_{2}\right)$ concentration in outer aqueous phase was selected as independent variables while the entrapment efficiency $\left(Y_{1}\right)$, particle size $\left(Y_{2}\right)$ and \% yield $\left(Y_{3}\right)$ were chosen as the dependent variables. The levels for these two parameters were determined from the preliminary trials. Furthermore, optimized formulation was evaluated for antimicrobial activity by evaluation of correlation between gentamicin concentrations obtained from actual loading and from inhibition diameters up to $24 \mathrm{~h}$. In vitro release profile of optimized formulation with and without additive in the outer aqueous phase was also studied.

\section{MATERIALS AND METHODS}

Materials Gentamicin sulphate (GM) (RS Spectra chemicals Ltd., Ahmednagar, Maharastra, India) and Eudragit RS100 (Rohm Pharma, Germany), polyvinyl alcohol (PVA) of molecular weight 30000 was from Loba chemicals, Mumbai, India, Pseudomonas aeruginosa MTCC 424 from 
IMTECH, Chandigarh, India. All other chemicals used in the study were of analytical grade.

Preparation and Characterization of the Microspheres (MS) GM loaded Eudragit RS100 microspheres were prepared by modified double emulsion solvent evaporation technique. ${ }^{17)}$ Briefly, GM (10 mg) dissolved in $0.2 \mathrm{ml}$ of a PVA $0.5 \%$ aqueous solution (W1) and $300 \mathrm{mg}$ of Eudragit RS100 dissolved in $5 \mathrm{ml}$ of dichloromethane (DCM) $(\mathrm{O})$ were mixed by mechanical stirring for $1 \mathrm{~min}(1500 \mathrm{rpm})$ to form a W1/O emulsion. This inner emulsion was then poured under vigorous stirring to $30 \mathrm{ml}$ of an aqueous solution (W2) containing $1 \% \mathrm{w} / \mathrm{v}$ of PVA with different proportion of additives using a magnetic stirrer for $2 \mathrm{~min}$. The resulting (W1/O/W2) solution was stirred for at least $3 \mathrm{~h}$ under room temperature (RT) to allow solvent evaporation and microspheres formation. After preparation, the microspheres were isolated by centrifugation $7000 \times \boldsymbol{g}$ for $10 \mathrm{~min}$, washed with distilled water and freezedried (Heto power dry LL 3000 Lyophilizer). The full factorial design and layout is shown in Table 1. The trials were performed in random order.

Particle Size and Morphology The particle size and size distribution of GM microspheres were measured by the method of laser light diffraction using a CILAS-1604 particle size analyzer (Cilas Inc., France). The particle size was expressed as volume mean diameter in micrometers. The surface morphology and shape were analyzed by scanning electron microscopy (Leo, VP-435, Cambridge, U.K.). Photomicrographs were observed at $300 \times$ and $500 \times$ magnification operated with an acceleration voltage of $15 \mathrm{kV}$ and working distance of $19 \mathrm{~mm}$ was maintained. Microspheres were mounted on the standard specimen mounting stubs and were coated with a thin layer $(20 \mathrm{~nm})$ of gold by sputter coater unit

Table 1. Full Factorial Experimental Design Layout with Coded Levels and Actual Values of Variables

\begin{tabular}{ccc}
\hline \hline Trial No. & $\begin{array}{c}\text { Variable } X_{1} \text { Glycerol } \\
\text { concentration }(\% \mathrm{v} / \mathrm{v})\end{array}$ & $\begin{array}{c}\text { Variable } X_{2} \mathrm{NaCl} \\
\text { concentration }(\% \mathrm{w} / \mathrm{v})\end{array}$ \\
\hline 1 & $20(-1)^{a)}$ & $3(-1)$ \\
2 & $60(+1)$ & $9(+1)$ \\
3 & $40(0)$ & $3(-1)$ \\
4 & $60(+1)$ & $3(-1)$ \\
5 & $20(-1)$ & $6(0)$ \\
6 & $40(0)$ & $6(0)$ \\
7 & $40(0)$ & $9(+1)$ \\
8 & $60(+1)$ & $6(0)$ \\
9 & $20(-1)$ & $9(+1)$ \\
\hline
\end{tabular}

a) Values in parentheses indicate coded levels of variables.
(VG Microtech, U.K.).

Entrapment Efficiency Twenty milligrams of the dried microspheres were accurately weighed and dissolved in DCM. $5 \mathrm{ml}$ of water is added to this, vortexed and shaken to extract the gentamicin to water phase.

The supernatant water is analyzed for gentamicin content using UV-Vis spectrophotometer (Shimadzu UV-1700, Pharmaspec, Tokyo, Japan). Derivatization is done with $o$-phthaldialdehyde and absorbance wavelength is $339 \mathrm{~nm}$ for UV-Vis spectrophotometer. ${ }^{18)}$ Results were expressed as mean ( \pm S.D.) of 3 experiments. The measured responses are shown in Table 2.

Factorial Design Traditional designing of pharmaceutical formulations are based on time consuming approach of changing one variable at a time which doesn't take into consideration the joint effects of independent variables. Thus, factorial design can serve as an essential tool to understand the complexity of pharmaceutical formulations. The results are expressed either as:

Simple linear

$$
\left(Y_{1}=b_{0}+b_{1} X_{1}+b_{2} X_{2}+b_{3} X_{3}\right)
$$

Or as second order polynomial equation

$$
\left(Y_{1}=b_{0}+b_{1} X_{1}+b_{2} X_{2}+b_{12} X_{1} X_{2}+b_{11} X_{1}^{2}+b_{22} X_{2}^{2}\right)
$$

Where, $b_{i}$ is the estimated coefficient for the factor $X_{i}$, while $Y_{i}$ is the measured response. The coefficients corresponding linear effects $\left(b_{1}\right.$ and $\left.b_{2}\right)$, interaction $\left(b_{12}\right)$ and the quadratic effects $\left(b_{11}\right.$ and $\left.b_{22}\right)$ were determined from the results of the experiment to identify the statistically significant terms. Reduced equation containing only statistically significant terms is then used to draw response surface plots (STAT-EASE, design expert, 7.0.3). To assess the reliability of the model, a comparison between the experimental and predicted values of the responses is also presented in Table 2 .

Bias was calculated by the following equation:

$$
\% \text { bias }=\frac{\text { predicted value }- \text { experimental value }}{\text { predicted value }} \times 100
$$

In Vitro Release Studies In vitro release of GM from microspheres was evaluated in Phosphate buffer ( $\mathrm{pH}$ 7.4). Triplicate samples of amount of microspheres equivalent to $20 \mathrm{mg}$ of GM were suspended in the prewarmed dissolution media $(20 \mathrm{ml})$ and maintained at $37^{\circ} \mathrm{C} \pm 0.5^{\circ} \mathrm{C}$ under stirring at $50 \mathrm{rpm}$. Samples were withdrawn at predetermined time intervals up to $24 \mathrm{~h}$ and the volume was replaced immedi-

\begin{tabular}{|c|c|c|c|c|c|c|c|c|c|}
\hline \multirow{2}{*}{ Serial No. } & \multicolumn{3}{|c|}{ Entrapment efficiency (\%) } & \multicolumn{3}{|c|}{ Size of microspheres $(\mu \mathrm{m})$} & \multicolumn{3}{|c|}{$\%$ Yield } \\
\hline & Actual & Pred & $\%$ Bias & Actual & Pred & $\%$ Bias & Actual & Pred & $\%$ Bias \\
\hline 1 & 34.82 & 34.74 & -0.230 & 60.43 & 61.16 & 1.193 & 79.9 & 80.60 & 0.868 \\
\hline 2 & 45.37 & 46.30 & 2.008 & 38.42 & 38.24 & -0.470 & 74.78 & 75.48 & 0.927 \\
\hline 3 & 30.64 & 29.66 & -0.033 & 56.32 & 58.30 & 3.39 & 91.20 & 88.24 & -3.354 \\
\hline 4 & 22.34 & 23.40 & 4.52 & 54.18 & 51.47 & -5.265 & 85.34 & 87.61 & 2.591 \\
\hline $5 *$ & 75.46 & 75.7 & 0.3828 & 46.47 & 42.47 & -9.418 & 100.3 & 100.48 & 0.179 \\
\hline 6 & 70.44 & 72.14 & 2.356 & 41.36 & 42.47 & 2.613 & 98.78 & 101.57 & 2.746 \\
\hline 7 & 50.32 & 49.60 & -1.452 & 42.44 & 39.35 & -7.852 & 89.05 & 89.22 & 0.190 \\
\hline 8 & 69.35 & 67.36 & -2.95 & 35.61 & 38.50 & 7.50 & 97.34 & 94.38 & -3.136 \\
\hline 9 & 51.94 & 51.73 & -0.405 & 33.24 & 36.50 & 8.931 & 95.56 & 94.69 & -0.918 \\
\hline
\end{tabular}
ately by fresh phosphate buffer. The samples withdrawn were

Table 2. Experimental and Predicted Responses Obtained for the Studied Parameters

Pred: Predicted. * Formulation with maximum entrapment. 
centrifuged ( $3000 \mathrm{rpm}, 15 \mathrm{~min}$, at room temperature). The supernatant water was analyzed for gentamicin content using UV-Vis spectrophotometer at absorbance wavelength of 339nm (Shimadzu UV-1700, Pharmaspec, Tokyo, Japan) by derivatization with $o$-phthaldialdehyde. ${ }^{18)}$ Results were expressed as mean ( \pm S.D.) of 3 experiments.

Antimicrobial Efficacy of Gentamicin Microspheres The GM released from Eudragit microspheres samples were tested for activity according to the Ph. Eur. Suppl. 2000 by the diffusion method 2.7.2 using $P$. aeruginosa as test organism. Firstly, aliquots of pure gentamicin at different concentrations were evaluated for inhibition diameters and calibration curve was plotted. The antimicrobial activity of drugloaded microspheres was evaluated by collecting samples (in vitro release aliquots) from the microspheres at different time intervals $(0.25,0.5,1,2,4,6,8,12,24 \mathrm{~h})$ and tested against P. aeruginosa.

Molten agar media was transferred to sterilized petridishes and allowed to solidify. The plates were swabbed with the culture of the microorganism. Wells equidistant from one another were made in the solidified medium using a sterilized well borer. The solutions $(100 \mu \mathrm{l})$ collected (in vitro drug release aliquots and pure drug solutions) were filtered through sterilized Millipore membrane filters $(0.45 \mu \mathrm{m})$ and carefully filled into the wells. Samples were allowed to diffuse for $2 \mathrm{~h}$ at room temperature. The plates were then incubated for $18 \mathrm{~h}$ at $37 \pm 0.5^{\circ} \mathrm{C}$. The diameter $(\mathrm{mm})$ of zone of growth inhibition surrounding each agar well was measured using a caliper. Concentration of GM obtained by inhibition diameters was then compared with the concentration obtained from release method to evaluate the effect of encapsulation on antimicrobial activity. Each experiment was carried out in triplicate.

\section{RESULTS AND DISCUSSION}

Microspheres with highly porous structure and large particle size were obtained in the absence of glycerol and $\mathrm{NaCl}$ in the external aqueous phase (Fig. 4). Moreover, microsphere yield and entrapment efficiency was relatively low with emulsifier alone. This could be due to rapid diffusion of hydrophilic drug into the continuous aqueous phase leading to decreased entrapment and rapid loss of drug. Glycerol concentration of $20,40,60 \% \mathrm{v} / \mathrm{v}$ and $\mathrm{NaCl}$ concentration of 3,6 , $9 \% \mathrm{w} / \mathrm{v}$ were selected from preliminary trials.

The entrapment efficiency $\left(Y_{1}\right)$, mean diameter $\left(Y_{2}\right)$ and $\%$ yield $\left(Y_{3}\right)$ from the nine experiments were used to generate predictor equations for Eudragit microspheres with independent variables as glycerol $\left(X_{1}\right)$ and $\mathrm{NaCl}\left(X_{2}\right)$ concentration in the outer aqueous phase.

All the formulations prepared within the experimental design layout yielded spherical microspheres. The results of multiple regression analysis and analysis of variance test (ANOVA) are summarized in Table 3.

The entrapment efficiency, mean diameter and \% yield of microspheres showed $R^{2}$ values of $0.9963,0.9224$ and 0.9484 respectively; indicating good fit and it was concluded that the quadratic statistical model adequately approximated the true surface.

The fitted model was:

$$
\begin{aligned}
& Y_{1}(\% \text { entrapment efficiency) } \\
& \quad=72.14-4.19 X_{1}+9.97 X_{2}+1.48 X_{1} X_{2}-0.59 X_{1}^{2}-32.51 X_{2}^{2} \\
& Y_{2}(\text { mean diameter }) \\
& \quad=42.47-1.99 X_{1}+9.47 X_{2}-2.86 X_{1} X_{2}-1.98 X_{1}^{2}+6.36 X_{2}^{2} \\
& Y_{3}(\% \text { yield })=101.47-3.05 X_{1}+0.49 X_{2}-6.55 X_{1} X_{2}-4.14 X_{1}^{2}-12.83 X_{2}^{2}
\end{aligned}
$$

The values of responses predicted from obtained model are shown in Table 2 along with the results obtained by experimentation. The close resemblances between observed and predicted response values in terms of low values of $\%$ Bias indicate the validity of the generated model.

For the entrapment efficiency $\left(Y_{1}\right)$ and \% yield $\left(Y_{3}\right)$ of microspheres, the calculated $F$ values of 162.23 , and 11.04 were found to be greater than the critical (table) value of $F_{5,3,95 \%}(9.01)$ whereas calculated $F$ value for mean diameter $\left(Y_{2}\right)$ was found to be 7.13 . Hence, it can be concluded that among the variables selected, one variable contributes significantly in the regression for measured $Y_{1}$ and $Y_{3}$ responses. Effect on $Y_{2}$ is relatively insignificant which indicates the involvement of other factors like stirring speed, polymer concentration, internal and external aqueous phase volume in regulating the size of microspheres. ${ }^{19)}$ Thus as expected from the estimated design, size of microspheres was not much dependent on the variables studied. The negative coefficient of $X_{1}$ in case of all responses studied refers to the low decrease in the response with subsequent increase in the concentration of glycerol $\left(X_{1}\right)$, whereas from the derived equation, $X_{2}$ exerts a positive response on entrapment $\left(Y_{1}\right)$, particle size $\left(Y_{2}\right)$ and $\%$ yield $\left(Y_{3}\right)$.

\begin{tabular}{|c|c|c|c|c|c|c|}
\hline \multirow{2}{*}{ Coefficients } & \multicolumn{2}{|c|}{$\%$ Entrapment efficiency } & \multicolumn{2}{|c|}{ Size of microspheres } & \multicolumn{2}{|c|}{$\%$ Yield } \\
\hline & FM & $\mathrm{RM}$ & FM & $\mathrm{RM}$ & FM & $\mathrm{RM}$ \\
\hline$b_{0}$ & 72.14 & 50.08 & 42.47 & 45.39 & 101.57 & 90.25 \\
\hline$b_{1}$ & -4.19 & -4.19 & -1.99 & -1.99 & -3.05 & -3.05 \\
\hline$b_{2}$ & 9.97 & 9.97 & -9.47 & -9.47 & 0.49 & 0.49 \\
\hline$b_{12}$ & 1.48 & - & 2.86 & - & -6.55 & - \\
\hline$b_{11}$ & -0.59 & - & -1.98 & - & -4.14 & - \\
\hline$b_{22}$ & -32.51 & - & 6.36 & - & -12.83 & - \\
\hline$R^{2}$ & 0.9963 & 0.2476 & 0.9224 & 0.7585 & 0.9484 & 0.0916 \\
\hline Significance & 0.0008 & 0.4260 & 0.0684 & 0.0141 & 0.0379 & 0.7496 \\
\hline$F$-value & 162.23 & 0.99 & 7.13 & 9.42 & 11.04 & 0.30 \\
\hline
\end{tabular}

The results indicate significant effect of variables $\left(X_{1}, X_{2}\right)$ and their interactions $\left(X_{1} X_{2}\right)$ on the measured responses. Reduced model shows insignificant value of $F$ for $Y_{1}$ and $Y_{3}$, but

Table 3. Regression Analysis Data for Measured Responses

FM: Full model. RM: Reduced model. 
significant in case of mean diameter of microspheres. These data indicate the significant role of interaction in controlling the size of microspheres and relatively small role of interaction between selected variables on the responses $\left(Y_{1}\right.$ and $\left.Y_{3}\right)$. This was further studied by studying the model in portions. ${ }^{20)}$

Response surface plot generated using Eqs. 4, 5, 6 are presented in Figs. $1-3$ respectively to observe the effects on the responses studied such as entrapment efficiency, size and \% yield of Eudragit-GM microspheres obtained by changing in-

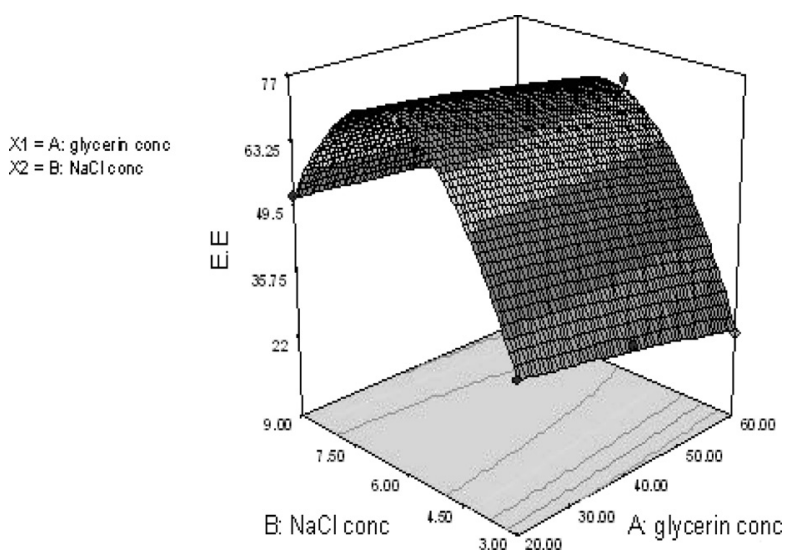

Fig. 1. Response Surface Curve for the Effect of Selected Variables on the Entrapment Efficiency of Microspheres

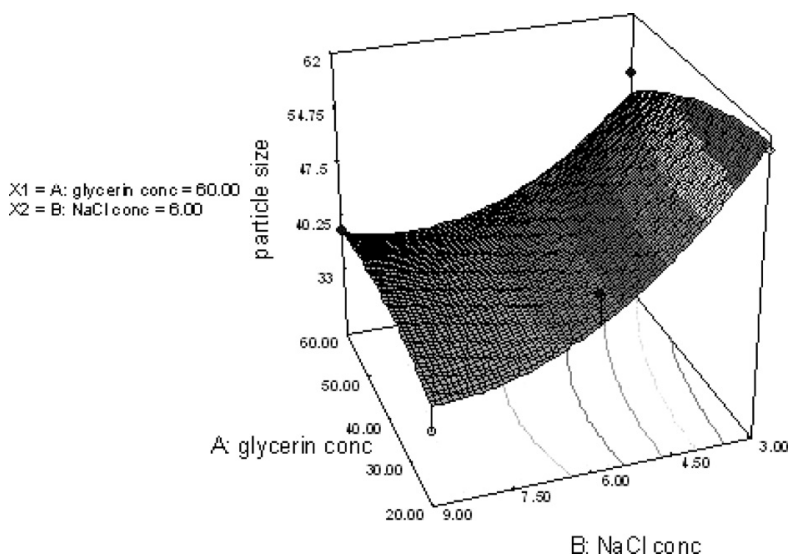

Fig. 2. Response Surface Curve for the Effect of Selected Variables on the Particle Size of Microspheres

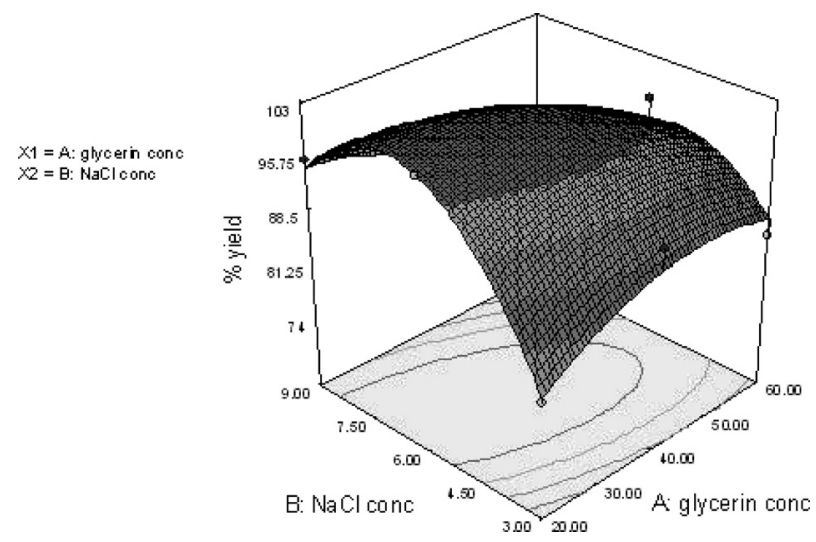

Fig. 3. Response Surface Curve for the Effect of Selected Variables on the $\%$ Yield of Microspheres dependent variables. The response surface plots (Figs. 1, 2) indicate the negative effect of external phase viscosity with increasing glycerol concentration on the responses studied. By keeping $\mathrm{NaCl}$ concentration at low level $\left(X_{2},-1\right)$ and glycerol concentration $\left(X_{1} ;-1,0,1\right)$ at different levels, viscosity of external aqueous phase increased from $2.5 \pm 0.24$ $\mathrm{mPa}, 4.75 \pm 0.48 \mathrm{mPa}$ to $8.32 \pm 0.47 \mathrm{mPa}$ respectively (Brookfield viscometer, LVDV-I+, U.S.A.) (Trial no. 1, 3, 4). The viscosity exerted negative effect on the average particle size and entrapment efficiency whereas \% yield was almost unchanged. Increase in viscosity of outer aqueous phase might contribute to reduction in particle size and a similar result was reported previously. ${ }^{21)}$ But decrease in particle size was not significant in present investigation might be due to interaction effect of both variables under study. Decrease in entrapment efficiency with increase in glycerol concentration can be related to partial dehydration of emulsifier leading to a deleterious effect on emulsification capability of the emulsifier molecule and hence, no improvement on microencapsulaion effect. ${ }^{22)}$ But in the absence of glycerol, highly clumped microspheres were obtained. Thus, minimum amount of glycerol was considered essential to obtain microspheres with decreased clumping necessitating little viscosity effect.

$\mathrm{NaCl}$ concentration in the outer aqueous phase generated

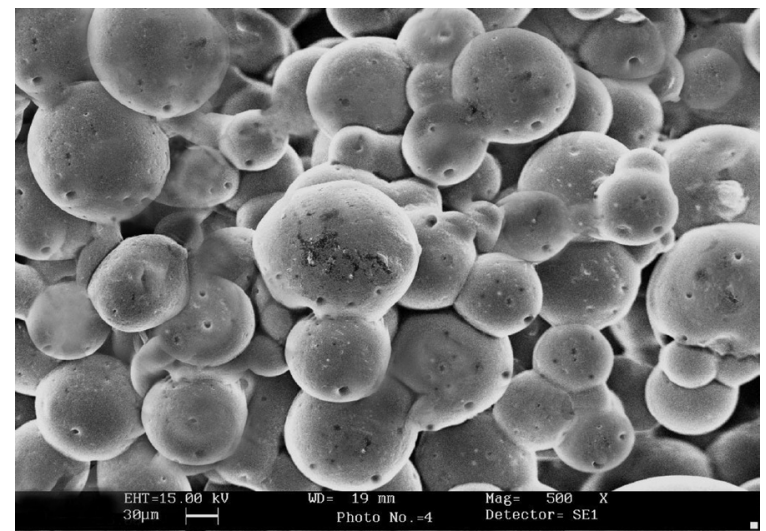

Fig. 4. Scanning Electron Micrograph of Microspheres with No Additives Indicating Microspheres with Large Size, Highly Porous Morphology and Fused Structures

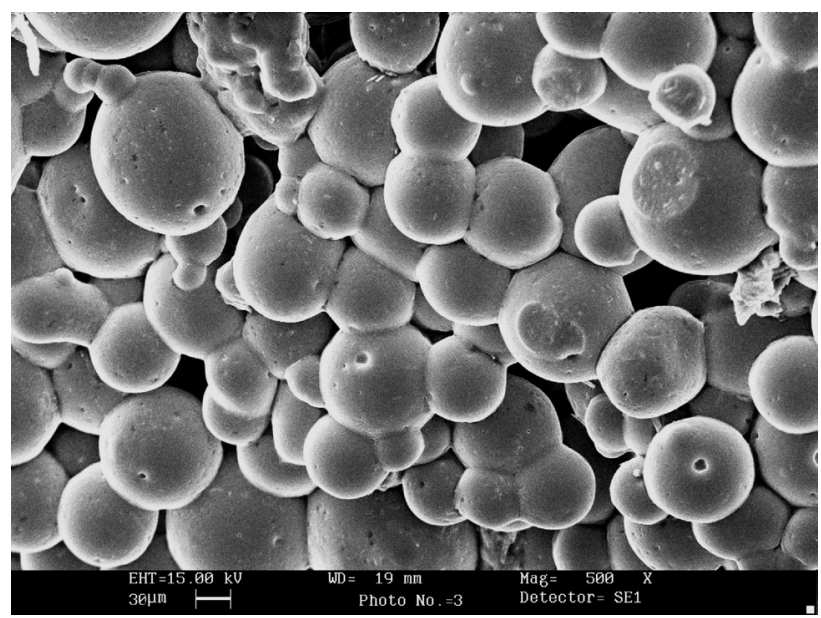

Fig. 5. Scanning Electron Micrograph of Microspheres with $3 \% \mathrm{w} / \mathrm{v} \mathrm{NaCl}$ Concentration Indicating Microspheres with Porous Morphology and Fused Structures (Trial no. 1) 
the positive effect on the entrapment efficiency up to a certain limit after which it showed the negative effect (Fig. 1). Improved entrapment efficiency could be related to microsphere surface characteristics as studied by Scanning Electron Micrography (SEM) under variable factor levels. Based on experimental and predicted values glycerol concentration $\left(X_{1}\right)$ was found to have insignificant effect on entrapment of the drug. So the morphology of microspheres with minimum level of $X_{1}\left(X_{1},-1\right)$ and different levels of $X_{2}(-1,0,1)$ were studied. Trial no. 1 microspheres $\left(X_{2},-1\right)$ with low levels of $\mathrm{NaCl}$ concentration demonstrated highly porous and fused structures (Fig. 5), trial no. $5\left(X_{2}, 0\right)$ demonstrated microspheres with smooth surface morphology exhibiting superior entrapment (Fig. 6) and trial no. $9\left(X_{2}, 1\right)$ microspheres exhibited smooth surface, but with fused characters leading to decreased entrapment and size (Fig. 7). Positive effect of $\mathrm{NaCl}$ concentration on the entrapment could be related to the osmotic pressure gradient existing across the inner and outer aqueous phase. The oil phase of a w/o/w double emulsion acts as a semi-permeable membrane and allows the diffusion of water across the organic phase when there is an osmotic pressure gradient across the inner and outer aqueous phase. This may either result in swelling or shrinkage of internal

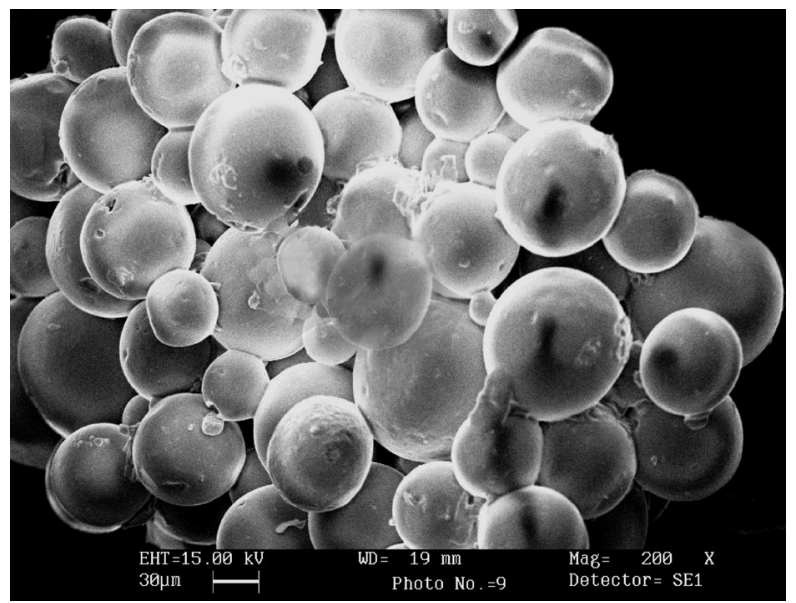

Fig. 6. Scanning Electron Micrograph of Microspheres with $6 \% \mathrm{w} / \mathrm{v} \mathrm{NaCl}$ Concentration Indicating Microspheres with Smooth Morphology (Trial no. 5)

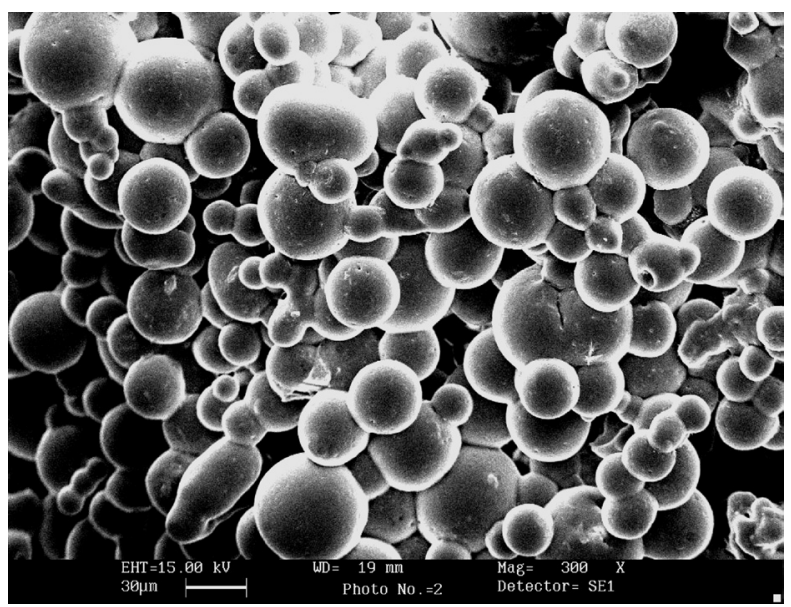

Fig. 7. Scanning Electron Micrograph of Microspheres with $9 \% \mathrm{w} / \mathrm{v} \mathrm{NaCl}$ Concentration Indicating Microspheres with Smooth Morphology but Fused Structures (Trial no. 9) droplets depending on the direction of osmotic gradient. ${ }^{23)}$

Forced by osmotic pressure gradient, water molecules in the outer aqueous phase might be diffused into the inner aqueous phase resulting in swelling of internal aqueous droplet. The w/o/w emulsion might be destabilized with increase in volume of inner aqueous phase and this might lead to poor entrapment of gentamicin. The water diffusion between two aqueous phases would be depressed gradually as $\mathrm{NaCl}$ concentration increased with improved microsphere yield and entrapment efficiency. $X_{2}$ exhibited positive effect on particle size of microspheres as supported by results of Zhang and Zhu. ${ }^{22)}$ Kassas also reported the similar increment in entrapment of $\mathrm{GM}$ by incorporation of $\mathrm{KCl}$ in outer aqueous phase due to the decrease in solubility of GM by salting out effect. $^{12)}$

$\mathrm{NaCl}$ at medium level $\left(X_{2}, 0\right)$ and Glycerol at low level $\left(X_{1},-1\right)$ yielded microspheres with highest drug content $(75.46 \%)$ and $\%$ yield $(100.3)$ with particle size in the range of $46.47 \pm 4.54 \mu \mathrm{m}$ may be due to combined effect of viscosity at low glycerol concentration and sufficient osmotic pressure gradient to suppress the diffusion of aqueous phases.

Response plots show that various combinations of $X_{1}$ and $X_{2}$ may satisfy any specific requirement (maximum drug entrapment and \% yield) while taking into consideration other factors such as cost, stability, etc. The results from the estimated ridge of maximum response in terms of desirability revealed that optimum glycerol $\left(X_{1}\right)$ and $\mathrm{NaCl}\left(X_{2}\right)$ concentrations were $20 \% \mathrm{v} / \mathrm{v}$ and $6 \% \mathrm{w} / \mathrm{v}$, respectively (trial no. 5) for desirable response.

To investigate the effect of outer aqueous phase concentration on the in vitro release behavior of microspheres, release tests of selected formulation (trial no. 5), microspheres with no additives and pure drug were carried out in phosphate buffer ( $\mathrm{pH}$ 7.4). Both microspheres exhibited almost similar release behavior but GM microspheres with additives exhibited a slight burst effect with release of $10.54 \pm 4.23 \%$ of GM in first hour followed by sustained release for up to $24 \mathrm{~h}$ whereas in case of microspheres with no additives in the external aqueous phase showed marked burst effect of 39.78土 $5.67 \%$ in the first hour followed by sustained release for $24 \mathrm{~h}$ (Fig. 8). Numbers of studies have been reported in the literature employing modification of the release of bioactives by inclusion of additives in the external aqueous phase. ${ }^{24,25)}$ $\mathrm{NaCl}$ and glycerol were selected as suitable additives that might act as physical barrier due to its high osmotic pressure and viscosity effect. Another possibility is that the $\mathrm{NaCl}$ competed with the surfactant for water molecules at the water/oil interface, generating a rigid interfacial layer that

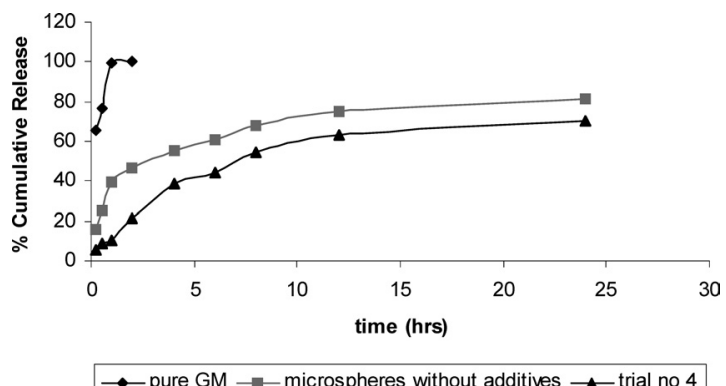

Fig. 8. In Vitro Release Profile of Gentamicin Sulphate Eudragit RS100 Microspheres (Trial no. 5) 


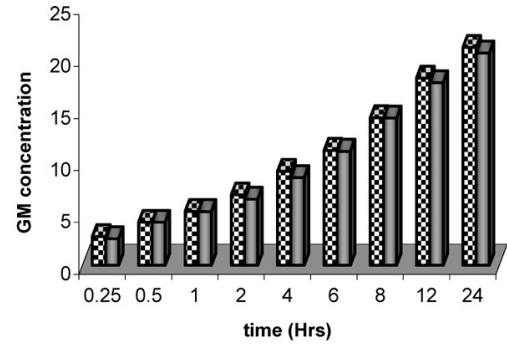

DGM Conc. From Actual Loading DGM Conc. From Inhibition Diameter

Fig. 9. Comparison of Concentration of GM as Determined from Antimicrobial Assay and Drug Release Studies

could be a more effective mechanical barrier to drug transfer with reduced burst. ${ }^{26)}$ Low burst effect in case of selected formulation might also be attributed to the fine difference in surface morphology, although no significant difference was observed due to the limited SEM resolution. The release mechanism of drug from microspheres (trial no. 5) was evaluated. A plot of 100-amount released $v s$. $\sqrt{t}$ for the formulation selected was linear with the value of coefficient of determination $\left(R^{2}\right)$ in Higuchi equation was found to be 0.9385 which indicates diffusion-controlled release, following Higuchi kinetics. ${ }^{27)}$

Evaluation of drug integrity is an important parameter as the exposure to organic solvents, interfaces, and stringent physical conditions of temperature and shear forces can lead to structural changes leading to loss of drug activity. ${ }^{28)}$ It is known that gentamicin sulphate is very stable in aqueous buffers over a wide $\mathrm{pH}$ and temperature range. ${ }^{9)}$ But, we conducted a bioassay, this demonstrated that the antimicrobial activity of the encapsulated gentamicin sulphate was fully preserved. The effects of microsphere encapsulation on the biological activity of the encapsulated drug were studied by an antibacterial assay by incubating the samples extracted at different time intervals from MS for $24 \mathrm{~h}$ at $37 \pm 0.5^{\circ} \mathrm{C}$. The assay measured growth inhibition of $P$. aeruginosa MTCC 424 on molten agar media. Inhibition diameter versus the log of GM concentration $(3-30 \mu \mathrm{g} / \mathrm{ml})$ of standard solutions closely correlated $\left(R^{2}-0.9786\right)$. The inhibition zones of MS incubation media corresponded almost similar to the GM concentrations expected from the amount of MS and the actual drug loading (Fig. 9). Thus, GM fully retained its biological activity upon encapsulation with negligible amount of loss may be due to processing steps during experimentation or adsorption to the vessel wall.

\section{CONCLUSION}

This study demonstrates the use of factorial design for the preparation of controlled release of gentamicin from Eudragit RS 100 microspheres with maximum loading and \% yield using double emulsion technique by variation in formulation parameters especially the composition of outer aqueous phase. This statistical technique allows scientists to examine more than one independent variable at a time. Increase in viscosity by introducing glycerol into outer phase didn't improve entrapment. However, microspheres with smooth surface, high yield and entrapment efficiency could be obtained by introducing sodium chloride into outer aqueous phase. It could be concluded from the above results that the osmotic pressure gradient between two aqueous phases should be considered during microencapsulation of hydrophilic drugs by double emulsion method to minimize the drug loss by diffusion in the outer aqueous phase. In vitro release tests indicated that microspheres prepared without additive exhibited marked burst effect due to porous surface morphology. Microencapsulation doesn't affect the bioactivity of entrapped drug as determined by antimicrobial assay. Further parameters can be identified by systemic approach for optimum formulation in terms of better long-term stability and to study the therapeutic effects of these particles in vivo.

Acknowledgements The authors are thankful to M/s RS spectra chemicals Ltd. Ahmednagar, Maharastra, India for the gift sample of Gentamicin sulphate; SIF, AIIMS, New Delhi, India, for Scanning electron micrography; Director, Institute of Pharmacy, Pt. Ravishankar Shukla University, Raipur (C.G.) India for providing all necessary facilities for carrying out this work, Chhattisgarh Council of Science and Technology (CCOST) and AICTE (under RPS) for financial assistance.

\section{REFERENCES}

1) Hunt T. K., Hopf H., Hussain Z., Adv. Skin Wound Care, 13, 6-11 (2000).

2) O’Meara S. M., Cullum N. A., Majid M., Sheldon T., British J. Surgery, 88, 4-21 (2002).

3) Hall W. H., Rev. Infect. Dis., 12, 1060-1090 (1990).

4) Gamazo C., Prior S., Irache J. M., Gander B., Díaz R., Vitas A. I., Recent Res. Dev. Antimicrob. Agents Chemother, 3, 59-82 (1999).

5) Tice T., Gilley R., J. Controlled Release, 2, 343-352 (1985).

6) Arshady R., Polym. Eng. Sci., 30, 915-924 (1990).

7) Wada R., Hyon S. H., Ikada Y., J. Controlled Release, 37, 151-160 (1995).

8) Ogawa Y., Yamamoto M., Okada H., Yashiki T., Shimamoto T., Chem. Pharm. Bull., 36, 1095-1103 (1988).

9) Rosenkrantz B. E., Greco J. R., Hoogerheide J. G., Oden M. E., "Analytical Profiles of Drug Substances," Vol. 9, ed. by Florey K., Academic Press, London, 1980 , pp. 295-340.

10) Mandal T., Tenjarla S., Int. J. Pharm., 137, 187-197 (1996).

11) El-Gibaldy I., Safwat S., Ahmed M., J. Microencapsulation, 13, 67 87 (1996).

12) Kassas R. A., J. Microencapsulation, 21, 71-81 (2004).

13) Shirakura O., Yamada M., Hashimoto M., Ishimaru S., Takayama K., Nagai T., Drug Dev. Ind. Pharm., 17, 471-483 (1991).

14) Govender S., Pillay V., Chetty D. J., Essack S. Y., Dangor C. M., Govender T., Int. J. Pharm., 306, 24-40 (2005).

15) Bhavsar M. D., Tiwari S. B., Amiji M. M., J. Controlled Release, 110, 422-430 (2006).

16) Dissemond J., Witthoff M., Brauns T. C., Haberer D., Goos M., Hautarzt, 54, 959-965 (2003).

17) Blanco-Príeto M. J., Leo E., Delie F., Gulik A., Couvreur P., Fattal E., Pharm. Res., 13, 1127-1129 (1996).

18) Robinson D. H., Sampath S. S., J. Pharm. Sci., 79, 428-431 (1991).

19) Dashora K., Saraf S., Saraf S., Pak. J. Pharm. Sci., 19, 177-181 (2006).

20) Mendenhall W., Sinoich T., "Multiple Regression. A Second Course in Business Statistics: Regression Analysis," 3rd ed., Dellen Publishing, California, 1989.

21) Scully D. B., J. Appl. Polym. Sci., 20, 2299 (1976).

22) Zhang J. X., Zhu K. J., J. Microencapsulation, 21, 775-785 (2004).

23) Florence A. T., Whitehill D., Int. J. Pharm., 11, 277-308 (1982).

24) Sansdrap P., Moës A. J., J. Microencapsulation, 15, 545-553 (1998)

25) Schaefer M. J., Singh J., AAPS PharmSciTech., 1(4), article 32 (2000).

26) Perugini P., Genta I., Conti B., Modena T., Pavanetto F., AAPS PharmSciTech., 2(3), article 10 (2001).

27) Dredan J., Antal I., Racz I., Int. J. Pharm., 145, 61-64 (1996).

28) Gander B., Merkle H. P., Nugyen V. P., Nam-tran H., J. Phys. Chem., 99, 16144-16148 (1995). 